\title{
Measurement and simulation of charge diffusion in a small-pixel charge-coupled device
}

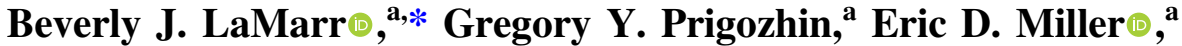 \\ Carolyn Thayer, ${ }^{\text {b }}$ Marshall W. Bautz $\odot,{ }^{\text {a }}$ Richard Foster $\odot,{ }^{a}$ \\ Catherine E. Grant $\odot,{ }^{a}$ Andrew Malonis, ${ }^{a}$ Barry E. Burke, \\ Michael Cooper, ${ }^{c}$ Kevan Donlon, ${ }^{c}$ and Christopher Leitz ${ }^{c}$ \\ ${ }^{a}$ Kavli Institute for Astrophysics and Space Research, Massachusetts Institute of Technology, \\ Cambridge, Massachusetts, United States \\ ${ }^{b}$ University of Michigan, Department of Climate and Space Sciences and Engineering, \\ Ann Arbor, Michigan, United States \\ 'Massachusetts Institute of Technology, Lincoln Laboratory, Lexington, Massachusetts, \\ United States
}

\begin{abstract}
Future high-resolution imaging x-ray observatories may require detectors with both fine spatial resolution and high quantum efficiency at relatively high x-ray energies $(E \geq 5 \mathrm{keV})$. A silicon imaging detector meeting these requirements will have a ratio of detector thickness to pixel size of six or more, roughly twice that of legacy imaging sensors. The larger aspect ratio of such a sensor's detection volume implies greater diffusion of x-ray-produced charge packets. We investigate consequences of this fact for sensor performance, reporting charge diffusion measurements in a fully depleted back-illuminated CCD with a thickness of $50 \mu \mathrm{m}$ and pixel size of $8 \mu \mathrm{m}$. We are able to measure the size distributions of charge packets produced by 5.9 and $1.25 \mathrm{keV} x$-rays in this device. We find that individual charge packets exhibit a Gaussian spatial distribution and determine the frequency distribution of event widths for a range of detector bias (and thus internal electric field strength) levels. At the largest bias, we find a standard deviation for the largest charge packets (produced by $x$-ray interactions closest to the entrance surface of the device) of $3.9 \mu \mathrm{m}$. We show that the shape of the event width distribution provides a clear indicator of full depletion and use a previously developed technique to infer the relationship between event width and interaction depth. We compare measured width distributions to simulations. Although we can obtain good agreement for a given detector bias, with our current simulation, we are unable to fit the data for the full range of bias levels with a single set of simulation parameters. We compare traditional, "sum-above-threshold" algorithms for individual event amplitude determination to Gaussian fitting of individual events and find that better spectroscopic performance is obtained with the former for $5.9 \mathrm{keV}$ events, whereas the two methods provide comparable results at $1.25 \mathrm{keV}$. The reasons for this difference are discussed. We point out the importance of read-noise driven charge detection thresholds in degrading spectral resolution, and note that the derived read noise requirements for mission concepts such as AXIS and Lynx are probably too lax to assure that spectral resolution requirements can be met. While the measurements reported here were made with a CCD, we note that they have implications for the performance of high aspect-ratio silicon active pixel sensors as well. () The Authors. Published by SPIE under a Creative Commons Attribution 4.0 International License. Distribution or reproduction of this work in whole or in part requires full attribution of the original publication, including its DOI. [DOI: 10.1117/1.JATIS.8.1.016004]
\end{abstract}

Keywords: x-ray image sensors; charge-coupled devices; sensor simulation.

Paper 21126 received Oct. 4, 2021; accepted for publication Jan. 14, 2022; published online Feb. $2,2022$.

\footnotetext{
*Address all correspondence to Beverly J. LaMarr, lamarr@mit.edu 


\section{Introduction}

Large format, megapixel solid-state image sensors have been mainstays of soft ( 0.1 to $10 \mathrm{keV})$ $\mathrm{X}$-ray astronomy for decades. ${ }^{1-6}$ These devices provide relatively large fields of view, more than adequate spatial resolution, and moderate energy resolution. Their imaging and spectroscopic capabilities also allow discrimination between x-rays from cosmic sources and unwanted background generated by charged particles encountered in the space environment. ${ }^{1-6}$

Future $x$-ray observatories are expected to require image sensors that exceed the capabilities of current flight detectors in a number of respects. For example, both the Lynx x-ray observatory, ${ }^{7}$ a large mission concept studied by NASA, and the smaller AXIS Probe-class concept, ${ }^{8}$ would require imaging detectors with better spatial resolution (pixel size $16 \mu \mathrm{m}$ ) than current systems, as well as excellent spectral resolution and quantum efficiency over a broader energy range $(0.2$ to $12 \mathrm{keV}$ ) (see Table 1$)$. Together these requirements dictate (for a silicon detector, at least) an unusually "tall" pixel, with pixel thickness to pixel width ratio $\sim 6: 1$, compared with current flight systems for which that ratio is roughly $\sim 3: 1$. Taller pixels entail greater lateral diffusion of the charge packets produced by absorption of x-ray photons, especially for x-rays at the low-energy extreme of the passband. These soft photons are absorbed very close to the detector entrance surface, so the charge packets they produce must drift farthest before collection and are therefore most likely to be shared among two or more adjacent pixels. Given the relatively small total quantity of charge in these packets, good detection efficiency and accurate spectroscopy at these energies requires low read noise. Detector noise requirements for these missions must account for this phenomenon, and accurate modeling of detector performance requires knowledge of it. This challenge faces all silicon $\mathrm{x}$-ray image sensors, including active pixel sensors of any architecture as well as CCDs.

Charge diffusion affects silicon sensor performance in other wavebands. In the visible and IR, its most important effect is a wavelength-dependent contribution to the system point spread function. ${ }^{9,10}$ In x-ray photon counting spectroscopic imaging applications, however, the effect of diffusion on spectral resolution and detection efficiency is of greater significance. We have been motivated to revisit this subject by the requirements of ambitious future X-ray observatories. The Lynx X-ray observatory, ${ }^{7}$ and even smaller-scale missions such as the AXIS Probe-class concept ${ }^{8}$ requires imaging detectors with moderate spatial resolution $(16 \mu \mathrm{m})$, but this must be accompanied by good response over a very broad energy range ( 0.3 to $10 \mathrm{keV})$. Together these requirements dictate (for a silicon detector, at least) an unusually "tall" pixel, with pixel size to thickness ratio $\sim 1: 6$. Critically, Lynx studies of the high-redshift universe also require excellent detection capability at the lowest energies. Design of detectors meeting these requirements requires careful optimization of diffusion and readout noise. This challenge faces all silicon image sensors, including active pixel sensors of any architecture as well as CCDs.

We note that other future developments in $\mathrm{x}$-ray astrophysics may also require a better understanding of charge diffusion in solid-state image sensors. These include $\mathrm{x}$-ray polarimetry ${ }^{11}$ and very high-resolution x-ray imaging. ${ }^{12}$

Table 1 Requirements for future x-ray probe and Flagship missions.

\begin{tabular}{lcc}
\hline \hline & \multicolumn{2}{c}{ Requirement } \\
\cline { 2 - 3 } Parameter & AXIS $^{8}$ Probe & Lynx $^{7}$ Flagship \\
\hline Angular resolution & \\
\cline { 2 - 3 } Energy range & $0.5^{\prime \prime}$ & $0.5^{\prime \prime}$ \\
Spectral resolution & 0.2 to $12 \mathrm{keV}$ & 0.2 to $10 \mathrm{keV}$ \\
Pixel size & $60 \mathrm{eV} \mathrm{FWHM} \mathrm{@1} \mathrm{keV}$ & $70 \mathrm{eV} \mathrm{FWHM} \mathrm{@} 0.3 \mathrm{keV}$ \\
Read noise & $16 \mu \mathrm{m}$ & $16 \mu \mathrm{m}$ \\
\hline \hline
\end{tabular}

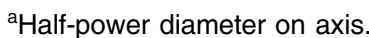

${ }^{\mathrm{b}}$ Full-width at half-maximum. 
We have been developing advanced CCDs for use in Lynx. ${ }^{13}$ CCDs are one of three types of imaging sensor, along with hybrid ${ }^{14,15}$ and monolithic ${ }^{16} \mathrm{CMOS}$ active pixel detectors, considered for this mission. Our work, which also has potential applications in probe-class, Explorer, and small missions, has focused on demonstrating fast, low-noise output amplifiers, low-power charge-transfer clocking, and development of appropriate application-specific circuits. ${ }^{17}$ One of the devices we have tested features a relatively small pixel $(8 \mu \mathrm{m})$ and a depletion thickness of $\sim 50 \mu \mathrm{m}$, and thus also offers the opportunity to evaluate the interplay of pixel size and charge diffusion in a sensor with a pixel thickness to width pixel-size ratio near that required for Lynx and AXIS. In this paper, we report measurements of charge packet size distributions as a function of device bias and x-ray energy obtained with this device. We compare these measurements to simulations made with a publicly available $\operatorname{code}^{18}$ developed to characterize the detectors of the Vera C. Rubin Observatory (VRO). ${ }^{19}$ We note that VRO CCDs are also high-aspect ratio devices and that $5.9-\mathrm{keV}$ x-ray characterization of these devices has been reported. ${ }^{20}$ Our results have implications for the soft $\mathrm{x}$-ray response of silicon active pixel sensors as well as CCDs.

\section{Data Acquisition and Analysis}

\subsection{Detector and X-Ray Sources}

Data reported here were obtained from a small-pixel, back-illuminated frame transfer detector developed at the MIT Lincoln Laboratory and designated CCID93. The device features $512 \times$ 512 pixels in both image and frame transfer areas. Pixels are $8 \mu \mathrm{m}$ wide and the device is $50 \mu \mathrm{m}$ thick. Its gate structure is implemented in a single layer of polysilicon with small inter-electrode gaps produced by photolithography. This configuration permits fast charge transfer with modest $(<3 \mathrm{~V})$ clock swings. ${ }^{13}$ The device architecture allows the substrate to be biased independently of the gate voltage and channel stop potentials to ensure that it is fully depleted. By varying the substrate bias voltage (hereafter " $V_{\text {sub" }}$ "), one can change the electric field strength in the depletion region and thus change the amount of lateral diffusion of $\mathrm{x}$-ray induced charge packets.

This test device was equipped with buried channel "trough" implants of varying widths, as well as a control region with no troughs. The trough implants provide significantly better charge transfer efficiency, and unless otherwise noted, we excluded data obtained in the trough-free region of the device. Details of the device architecture are described elsewhere. ${ }^{13}$

We operated the detector at a temperature of $-50^{\circ} \mathrm{C}$ with serial and parallel register rates of $2.0 \mathrm{MHz}$ and $500 \mathrm{kHz}$, respectively, and gate voltage swings of $3.0 \mathrm{~V}$ peak to peak. Readout noise is typically 4.5 electrons, RMS, under these conditions. An STA Archon commercial controller ${ }^{21}$ was used to operate the detector and digitize the data.

Data were collected in x-ray photon-counting mode using either a radioactive ${ }^{55} \mathrm{Fe}$ source (producing Mn K x-rays at 5.9 and $6.4 \mathrm{keV}$ ) or a grating monochromator fed by an electronimpact source at energies below $2 \mathrm{keV}$. The spectral resolving power of the monochromator is typically $\lambda / \Delta \lambda=E / \Delta E \sim 60-80$, far exceeding that of the detector at the energies of interest. In this work, we focus on results at two energies, 5.9 and $1.25 \mathrm{keV}$ from $\mathrm{Mn} \mathrm{K} \alpha$ and $\mathrm{Mg} \mathrm{K} \alpha$ photons, respectively.

\subsection{Data Acquisition}

Data were obtained for each energy and substrate bias value of interest by repeatedly reading the detector, storing each full-frame readout as a distinct file. The exposure time for each frame was $0.15 \mathrm{~s}$, and source flux was adjusted to less than $\sim 25$ detected photons (events) per frame $\left(\lesssim 10^{-4}\right.$ events per pixel) to minimize pileup. Approximately $2.3 \times 10^{5}$ events were collected for each configuration. Data were obtained at seven values of $V_{\text {sub }}$ ranging from -0.2 to $-20 \mathrm{~V}$.

\subsection{Analysis Methods}

Data were acquired in groups of 100 consecutive frames. From each acquired frame, an average of the overclock region was subtracted to remove drift of the DC level. A bias (zero-signal) frame was then computed for each group using a clipped average algorithm to remove signal from 
$\mathrm{x}$-rays and cosmic rays. The bias frame was subtracted pixel-by-pixel from each data frame in its group. X-ray events were then identified by searching the bias-subtracted frames for pixels with amplitudes exceeding a fixed threshold (the event threshold), which are also local maxima. The event threshold was set to 10 times the RMS noise level for $5.9 \mathrm{keV}$ x-rays and 7.5 times the noise for $1.25 \mathrm{keV}$. This level is high enough to reject spurious events due to noise while low enough to accept evenly split $x$-rays. For each event, the values of a $7 \times 7$ pixel array centered on the located maximum were recorded in an event list.

We found that in this relatively small pixel device almost all of the events have signal charge spread over multiple pixels. To characterize the signal distribution, we $\mathrm{fit}^{22}$ a two-dimensional (2D) Gaussian function to the pixel values of each event. In these fits, the Gaussian standard deviation $\left(\sigma_{\text {spatial }}\right)$ was constrained to be the same in both dimensions, and a constant additive term was included to allow for a local offset bias level. In this way, we obtained five parameters (two for position, plus one each for amplitude, $\sigma$, and bias offset) for each event.

For comparison, we also computed the sum of all the pixel values in a seven by seven pixel island above a second, lower, threshold value, known as the "split event" threshold. This is the event energy reconstruction method in use for all past and current x-ray CCD missions for astrophysics but with the number of pixels in each island increased to compensate for the much smaller pixels in our device. We set the split threshold at four times the RMS readout noise per pixel.

\subsection{Simulations}

Simulated event lists were constructed using POISSON CCD,${ }^{18}$ a software package that, given the structural and operational characteristics of the detector, (1) solves Poisson's equation for the electric field in three dimensions at all points within the detector volume and (2) tracks the drift and diffusion of electrons placed within this volume until they reach the buried channel, where they are collected in pixels defined by the channel stop and barrier gate fields. We set up the simulated detector to reflect as nearly as possible the characteristics and operating conditions of the CCID93 device described in Sec. 2.1: $50 \mu \mathrm{m}$ thickness, $8 \mu \mathrm{m}$ pixels, and three-phase gate structure with one collecting gate held at $+1.5 \mathrm{~V}$ and two barrier gates held at $-1.5 \mathrm{~V}$. Implant and doping parameters were provided by MIT Lincoln Laboratory. The simulation volume covered $9 \times 9$ pixels, with nonlinear grid spacing in the vertical direction allowing finer grid sampling of $15 \mathrm{~nm}$ at the front and back sides of the device to properly capture the electric field structure, and coarser grid sampling (up to $150 \mathrm{~nm}$ ) throughout the bulk of the device. The electric field was simulated at an operating temperature of $-50^{\circ} \mathrm{C}$ at each of the $V_{\text {sub }}$ settings used for the real device.

For each $V_{\text {sub }}$, we simulated detection of 200,000 5.9 and $1.25 \mathrm{keV}$ photons by introducing small clouds of electrons and allowing them to drift and diffuse until collected in pixels. The number of electrons in each cloud was drawn from a Fano noise distribution appropriate for the photon energy in $\mathrm{Si}$ (e.g., $1615 \pm 14$ electrons for $5.9 \mathrm{keV}$ ), and the interaction depth was drawn from an exponential distribution with appropriate attenuation length. Each interaction location was generated from a uniform distribution across the central pixel of the $9 \times 9$-pixel simulation volume. The final pixel distribution of electrons was converted into an event island in energy units. Simulated Gaussian readout noise with RMS of 4.5 electrons was added to each pixel, and event detection and characterization were performed in the same way as the lab data.

The default charge diffusion parameters in Poisson CCD produced much more diffusion in the simulations than we observe in the lab data, with much larger event sizes. Since the purpose of the simulations was to illuminate the physical processes responsible for the observed data, we tuned the DiffMultiplier parameter so the simulated event size distributions closely matched the real data, as described in the following section. These DiffMultiplier values ranged from 1.4 to 1.7 for $V_{\text {sub }}=-0.2$ to $-20 \mathrm{~V}$, compared with the value of 2.3 determined from charge diffusion measurements in Vera Rubin Observatory CCDs. ${ }^{18}$ This parameter is implemented in Poisson CCD as a scale factor for the charge carrier thermal velocity, given by $v_{\text {th }}=\left(8 k T / m_{e} \pi\right)^{1 / 2} \times$ DiffMultiplier, ${ }^{18}$ where $m_{e}$ is the bare electron mass. In effect, it specifies a value for the thermal velocity effective mass of the electron in the Si conduction band, $m_{e, t c}^{*}$. Previous estimates suggest $m_{e, t c}^{*} / m e \approx 0.27-0.28,{ }^{23}$ which would indicate DiffMultiplier $\approx 1.9$ as an appropriate 
value. The modest difference between that and the value DiffMultiplier $=1.7$ required to match our data at highly negative $V_{\text {sub }}$ (fully depleted substrate) is likely due to the limitations of the carrier transport model used in Poisson CCD. ${ }^{18}$ The larger differences at less negative $V_{\text {sub }}$ could arise from incorrect treatment of undepleted bulk in the simulator (C. Lage, private communication). We saw no difference in the amount of diffusion if electron cloud Coulomb repulsion was turned on (FE55 mode). These issues will be explored in a future paper aimed at further validating the simulations and producing a higher-fidelity simulation methodology for these thick, small-pixel x-ray CCDs. We will also consider implementing techniques used to model drift and diffusion developed recently by other groups. ${ }^{24,25}$

\section{Measurements}

\subsection{Overview}

We measure (2D) position, amplitude, and width (2D Gaussian $\sigma_{\text {spatial }}$ ) of each event as described in Sec. 2.3. Figure 1 shows scatter plots of summed-pixel event amplitude versus event width for both x-ray energies and three different values of internal detector bias $V_{\text {sub. }}$. Here, color indicates density of events in the amplitude-width plane, and projections of the data in these two axes show the corresponding distributions in amplitude and width. Approximately 230,000 events are represented in each panel of the figure.

The (vertical) amplitude distributions show the detector's spectral response function at energies of 5.9 and $6.4 \mathrm{keV}$ (upper panels) and $1.25 \mathrm{keV}$ (lower panels). Silicon K-escape and fluorescence lines are evident in the upper panels. The (horizontal) width distributions reflect the amount of lateral diffusion experienced by charge packets before collection.

Comparison of the three columns in Fig. 1 shows how the device response changes with the strength of the electric fields in the detector's photosensitive volume, with the strongest field $\left(V_{\text {sub }}=-20 \mathrm{~V}\right)$ on the left and the weakest $\left(V_{\text {sub }}=-1 \mathrm{~V}\right)$ on the right. Thus, in the left column, the relatively strong fields provide relatively rapid charge collection, and thus relatively small event widths, as well as relatively good spectral (amplitude) resolution. As field strength

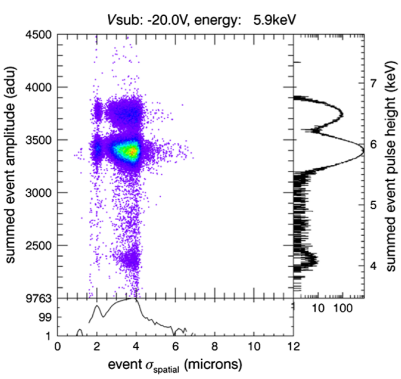

(a) $5.9 \mathrm{keV}, v_{\text {sub }}=-20 \mathrm{~V}$

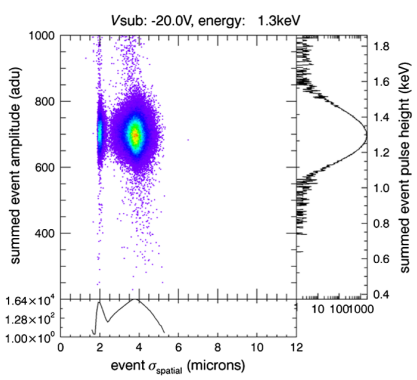

(d) $1.25 \mathrm{keV}, V_{\text {sub }}=-20 \mathrm{~V}$

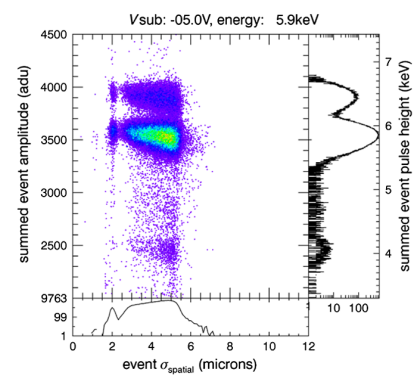

(b) $5.9 \mathrm{keV}, v_{\text {sub }}=-5 \mathrm{~V}$

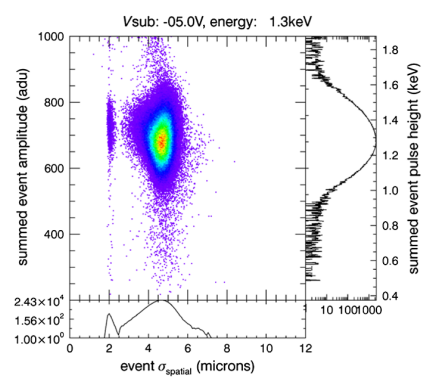

(e) $1.25 \mathrm{keV}, v_{\text {sub }}=-5 \mathrm{~V}$

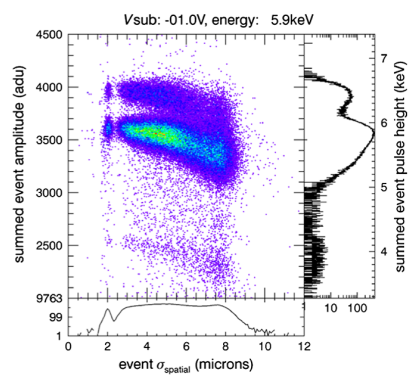

(c) $5.9 \mathrm{keV}, v_{\text {sub }}=-1 \mathrm{~V}$

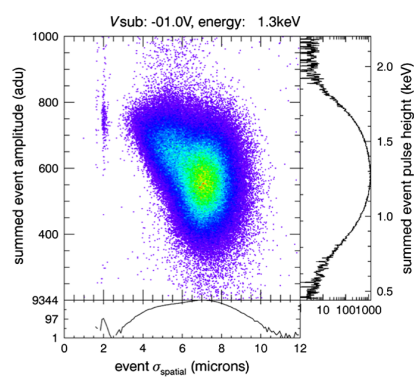

(f) $1.25 \mathrm{keV}, v_{\text {sub }}=-1 \mathrm{~V}$

Fig. 1 Scatter plots of summed-pixel amplitude versus width (2D Gaussian $\sigma$ ) for $\mathrm{x}$-ray events at two energies and three values of the substrate bias $\left(V_{\text {sub }}\right)$. The color indicates the areal density of events in the width/amplitude plane. Histograms of event amplitude and width are projected onto the right and bottom edges, respectively, of each panel. 


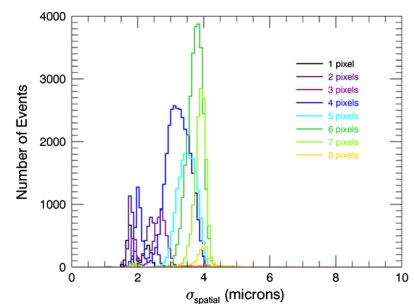

(a) $5.9 \mathrm{keV}, v_{\text {sub }}=-20 \mathrm{~V}$

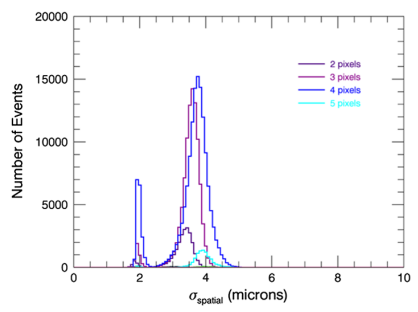

(d) $1.25 \mathrm{keV}, v_{\text {sub }}=-20 \mathrm{~V}$

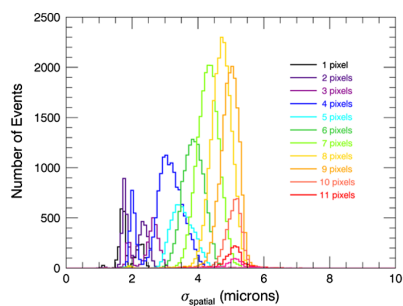

(b) $5.9 \mathrm{keV}, v_{\text {sub }}=-5 \mathrm{~V}$

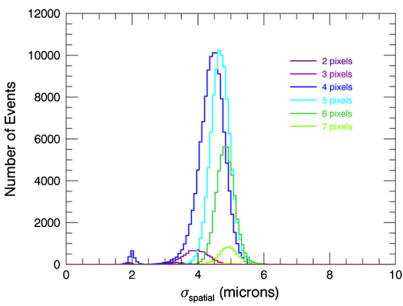

(e) $1.25 \mathrm{keV}, v_{\text {sub }}=-5 \mathrm{~V}$

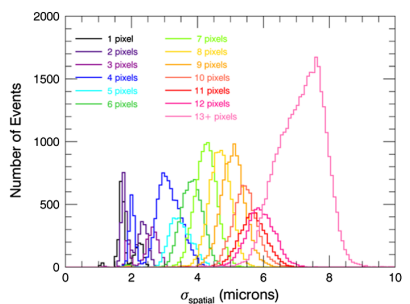

(c) $5.9 \mathrm{keV}, v_{\text {sub }}=-1 \mathrm{~V}$

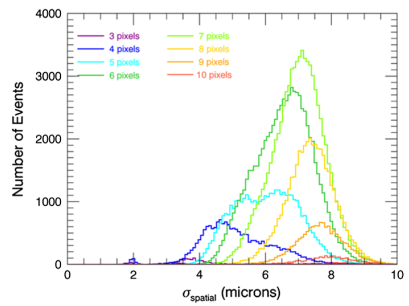

(f) $1.25 \mathrm{keV}, V_{\text {sub }}=-1 \mathrm{~V}$

Fig. 2 Histograms of fitted event width (Gaussian $\sigma_{\text {spatial }}$ ) by number of pixels above the split threshold (pixel multiplicity) for various values of the substrate bias $V_{\text {sub }}$ and for two x-ray energies.

decreases in the center and right columns, the figures show the effects of progressively smaller fields ( $V_{\text {sub }}=-5 \mathrm{~V}$ and $-1 \mathrm{~V}$, respectively): both width and amplitude distributions become progressively wider. In fact, the double-peaked width distribution in Fig. 1(c) suggests that the detector is no longer fully depleted at this low value of substrate bias. Events with the largest widths at this value of $V_{\text {sub }}$ also show depressed amplitude, implying that events produced in the undepleted region of the detector suffer from incomplete charge collection as well as large lateral diffusion. $^{26,27}$

A closer look at the spatial width distributions is shown in Fig. 2, which shows distributions for events comprising various number of pixels above the split threshold. (Hereafter, we denote the latter quantity as "pixel multiplicity"). The panels in this figure correspond to those in Fig. 1.

Comparison of the upper and lower panels of Fig. 2 shows the marked energy dependence in the size distributions. In general, events with a given pixel multiplicity are spatially smaller at the higher energy. In addition, for $5.9 \mathrm{keV}$ events, there is a clear correlation between width and number of pixels above threshold, whereas at $1.25 \mathrm{keV}$ the variation in size with number of pixels is much smaller. Finally, at the stronger fields $\left(V_{\text {sub }} \leq-5 \mathrm{~V}\right)$, the maximum size of events is similar at both energies (about $4 \mu \mathrm{m}$ for $V_{\text {sub }}=-20 \mathrm{~V}$ ).

These observations are straightforward consequences of the expected energy dependence of $\mathrm{x}$-ray interaction depths in the detector, coupled with the dependence of lateral diffusion on interaction depth. The relatively more penetrating $5.9 \mathrm{keV}$ x-rays (attenuation length in silicon $\sim 29 \mu \mathrm{m})$ are absorbed throughout the $50 \mu \mathrm{m}$ thickness of the detector. The $1.25-\mathrm{keV}$ x-rays (attenuation length $5 \mu \mathrm{m}$ ) all interact in the third of the detector closest to the entrance window. As a result, there is a much larger range of lateral diffusion and therefore sizes in the population of $5.9 \mathrm{keV}$ events. An important conclusion from this figure, however, is that when the detector is fully depleted (two left columns), the maximum amount of diffusion is the same at both energies.

In all cases, the histogram peaks near $\sigma=2 \mu \mathrm{m}$ are associated with events for which charge is detected in four or fewer pixels. Although the amplitude of these events can be measured accurately by summing the pixels above split threshold, their sizes cannot be resolved by our detector or reliably measured using the Gaussian fitting algorithm. Since our pixel size is comparable to or greater than the intrinsic (prepixelization) size of the charge packets, our best-fit values of $\sigma_{\text {spatial }}$ are biased high relative to those of the intrinsic distributions. The magnitude of this bias depends on the intrinsic width. Analytic calculations suggest, and simulations confirm, that this bias ranges from more than $30 \%$ to less than $10 \%$ for $3 \mu \mathrm{m} \lesssim \sigma \lesssim 6 \mu \mathrm{m}$. For $\sigma<3 \mu \mathrm{m}$, our fitting algorithm fails to converge and tends to return values of $\sigma \approx 2 \mu \mathrm{m}$. This accounts for the small peaks at this value in the width distributions shown in Figs. 1 and 2. 
Such spatially unresolved charge distributions can arise in different ways. At $5.9 \mathrm{keV}$, some $\mathrm{x}$-rays can penetrate deep into the detector to interact close to the CCD's buried channel and thus can produce events that suffer very little diffusion. After pixelization, all information about the intrinsic size of these events is lost and the Gaussian model does not describe them well. At $1.25 \mathrm{keV}$, all events must be subject to considerable diffusion. In this case, however, the relatively large spatial extent of the charge distribution, coupled with the relatively small total number of electrons, can produce low signal-to-noise ratios in the peripheral pixels. Simulations confirm that fits may not converge in these circumstances.

\subsection{Measurement and Applications of the Event Charge Distribution}

The data presented in the previous section illustrate the broad range of event sizes that occur even under monochromatic x-ray illumination. A complete characterization of detector response at even a single energy thus requires knowledge of both the distribution of charge for events of a given width (which we have assumed to be Gaussian in the foregoing), and the distribution of event widths. Previous work by Prigozhin and co-workers ${ }^{26}$ has shown how this problem can be addressed by direct measurement.

Briefly, we can exploit the fact that for a monochromatic incident beam, both the number of $\mathrm{X}$-rays absorbed and the magnitude of lateral diffusion are monotonic functions of the depth in the detector at which the X-ray is absorbed. The former relation is simply a consequence of the attenuation of the X-ray intensity with depth and can be determined reliably from known material properties. The latter results because the time required for the liberated electrons to drift from the interaction point to the buried channel, and thus the amount of lateral diffusion, decreases with depth. The expected width-depth relation is illustrated in Fig. 3, which shows simulation results for $5.9 \mathrm{keV} \mathrm{X}$-rays for various values of $\mathrm{V}_{\text {sub }}$. Because (for a given field configuration) this relationship is also monotonic in depth, it is possible to measure it using X-ray data. Moreover, it is also possible to obtain a high-quality measurement of the shape of the charge distribution for events interacting at a given depth and thus test our assumption that this distribution is Gaussian.

\subsubsection{Average charge distribution at fixed depth}

To characterize event charge distributions at fixed depth, we first order events by their measured values of $\sigma_{\text {spatial }}$, and then select 1000 events surrounding each of several percentile points in the

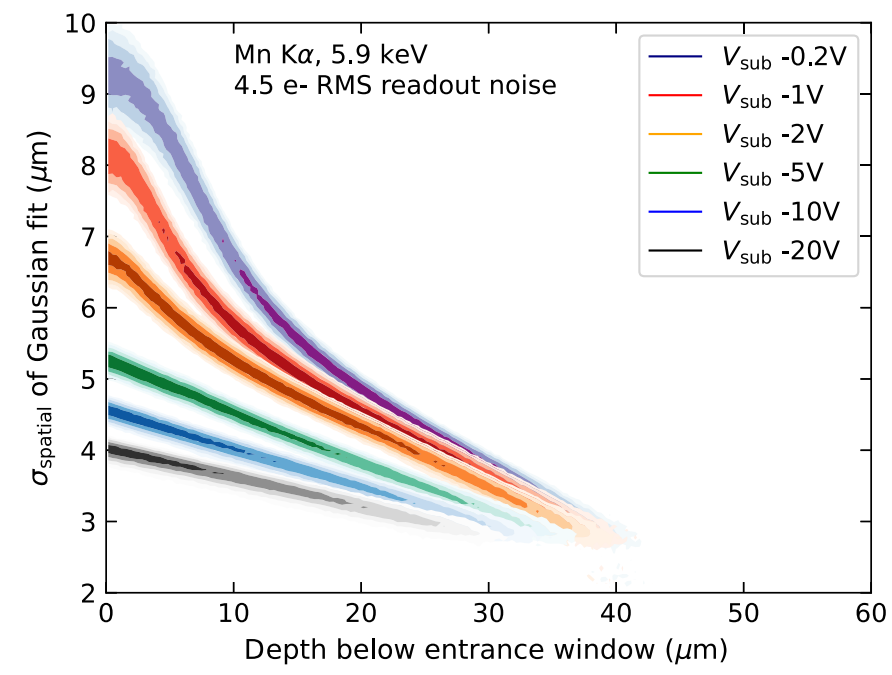

Fig. 3 Simulated best-fit Gaussian $\sigma_{\text {spatial }}$ as a function of the photon interaction depth for different values of substrate bias parameter $V_{\text {sub }}$ and photon energy of $5.9 \mathrm{keV}$. The device is $50 \mu \mathrm{m}$ thick. Contours show $5 \%, 10 \%, 20 \%, 40 \%$, and $70 \%$ of the maximum density of points for each $V_{\text {sub. }}$. There is a clearly monotonic trend of event width with depth. It is also clear that the maximum event size depends on $V_{\text {sub }}$. 
cumulative $\sigma_{\text {spatial }}$ distribution, thus selecting a group of events coming from approximately the same depth in the device. To minimize the effects of pixelization, we create a $70 \times 70$ subpixel grid over the $7 \times 7$ pixel island around each event's central pixel. Each element of the subpixel grid was assigned an amplitude equal to $1 / 100$ of the amplitude of the pixel in which that element lies. We next align the centroid of each event with the origin of the subpixel grid, and then average all subpixel amplitudes over all events in the percentile group. Since centroids can be determined with precision finer than a single pixel, this procedure produces a mean event charge distribution with subpixel resolution.

The results for $5.9 \mathrm{keV}$ at three different percentiles and three different values of $\mathrm{V}_{\text {sub }}$ are shown in Fig. 4. Each row corresponds to a different value of $V_{\text {sub }}$, with the strongest field at the top. Each column is a different value of percentile in the cumulative width distribution, with the largest percentile width at the left.

Qualitatively, the figure shows, as expected, that for a given field distribution, event widths become smaller as the interaction depth increases (to the right), and that for a given depth, event widths also become smaller as the field strength increases (bottom to top). Note that at $\mathrm{V}_{\text {sub }}=-1 \mathrm{~V}$ (bottom row) the detector is only partially depleted, and the cloud size is quite large. In both the middle and top rows, with more negative $\mathrm{V}_{\text {sub }}$, the device is fully depleted and cloud size shrinks accordingly. As noted in Sec. 3.1, at very small cloud sizes $\left(\sigma_{\text {spatial }}<4 \mu \mathrm{m}\right)$, the effects of pixelization become evident: the inferred shape tends to become non-circular, reflecting that of the pixels rather than that of the intrinsic charge distribution. We shall return to the consequences of this under-sampling in Sec. 3.3 below.

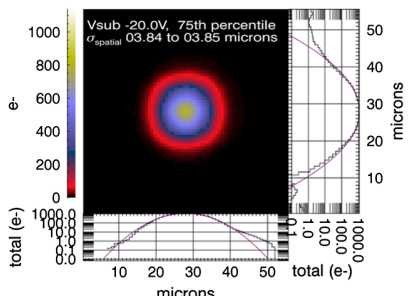

(a) $\sigma_{\text {spatial }}=3.8 \mu \mathrm{m}$

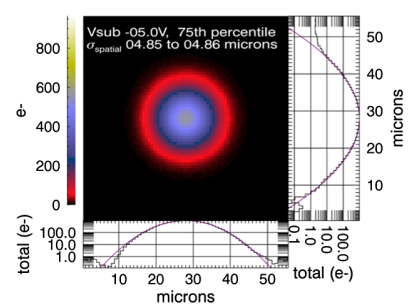

(d) $\sigma_{\text {spatial }}=4.9 \mu \mathrm{m}$

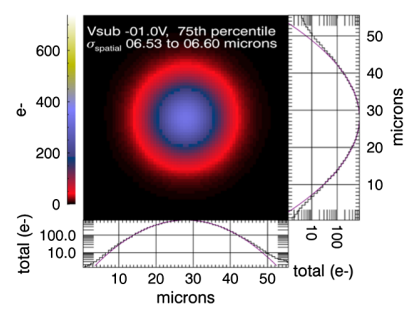

(g) $\sigma_{\text {spatial }}=6.5 \mu \mathrm{m}$

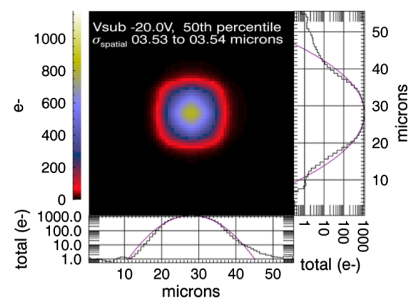

(b) $\sigma_{\text {spatial }}=3.5 \mu \mathrm{m}$

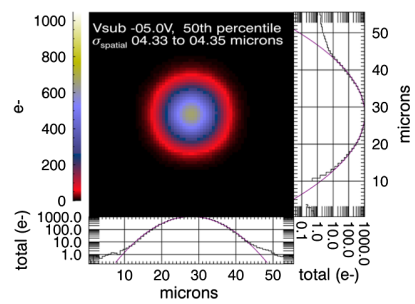

(e) $\sigma_{\text {spatial }}=4.3 \mu \mathrm{m}$

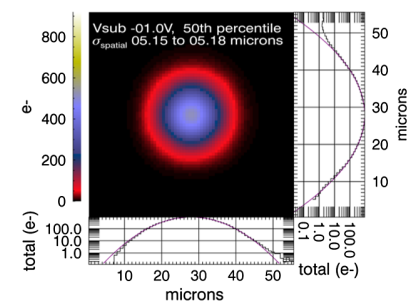

(h) $\sigma_{\text {spatial }}=5.2 \mu \mathrm{m}$

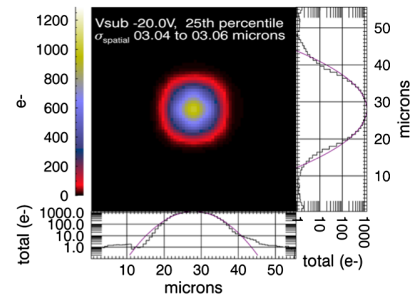

(c) $\sigma_{\text {spatial }}=3.0 \mu \mathrm{m}$

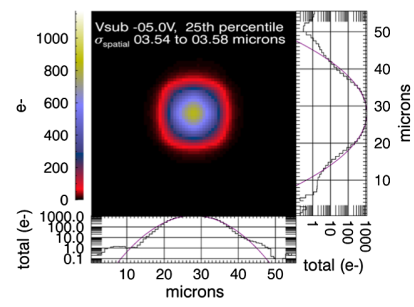

(f) $\sigma_{\text {spatial }}=3.5 \mu \mathrm{m}$

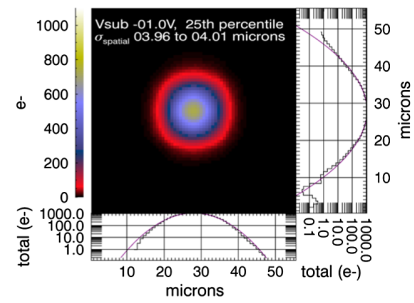

(i) $\sigma_{\text {spatial }}=4.0 \mu \mathrm{m}$

Fig. 4 Measured average event profiles produced by $5.9 \mathrm{keV} x$-rays. The top, middle, and bottom rows show results for $V_{\text {sub }}=-20 \mathrm{~V},-5 \mathrm{~V}$, and $-1 \mathrm{~V}$, respectively. The left, middle, and right columns are for events in the 75th, 50th, and 25th width percentiles, respectively. Horizontal and vertical projections of the $2 \mathrm{D}$ distributions, together with best-fit Gaussians, are shown for each case. Here, the units of ordinates are electrons per pixel. 
How accurate is our assumption of a Gaussian charge distribution? Projections (i.e., sums along rows and columns) of the signal distribution are shown to the right and beneath each panel in Fig. 4, along with best-fit Gaussian curves. The central portions of the profiles are clearly very well described by a Gaussian function over at least two orders of magnitude, which is consistent with theoretical calculations for clouds formed within the depleted region. ${ }^{28}$ There is a slight excess in measured signal compared to the Gaussian wings, but the level of discrepancy is on the order of $0.1 \%$ of the total charge packet signal per pixel. We note that the deviation is largest for the data with the smallest $\sigma_{\text {spatial }}$, which are most affected by the pixelization. We also note that for vertical signal distributions the tail on the top side is noticeably higher than on the bottom side, no doubt as a result of charge transfer inefficiency that causes electrons trapped during parallel transfer to be re-emitted into the pixels behind the event center. A similar distortion of charge-cloud shape has been used previously as a diagnostic of charge transfer inefficiency in electron multiplier CCDs. ${ }^{29}$ We conclude that charge packets are Gaussian to a very good approximation and that the most significant deviations arise at small widths as a result of pixelization.

\subsubsection{Inferring interaction depth from the cumulative event width distribution}

As noted at the beginning of this section, since both the density of X-ray events and the size of the corresponding charge distributions vary monotonically with interaction depth, it is possible to map the density of the event widths onto the distance from the illuminated surface. This idea was proposed previously by Prigozhin et al. ${ }^{26} \mathrm{We}$ implement this map as follows. The integral number of photons absorbed between the illuminated detector surface and the plane at depth $z$ below that surface can be written $N_{z}=N_{0}(1-\exp (-z / \lambda))$. Here, $N_{0}$ is the incident photon fluence. The total number of events $N_{\text {total }}$ absorbed in an ideal device with thickness $t$ is $N_{\text {total }}=N_{0}(1-\exp (-t / \lambda))$, so the cumulative fraction of events absorbed above depth $z$, $N_{z_{-} \text {frac }}=N_{z} / N_{\text {total }}$, is

$$
N_{z_{\text {frac }}}=\frac{1-\exp (-z / \lambda)}{1-\exp (-t / \lambda)}
$$

This relationship is monotonic and can therefore be inverted to give the value of $z$ as the function of the fraction of events above that depth:

$$
z=-\lambda \ln \left(1-N_{z_{-} \text {frac }}(1-\exp (-t / z))\right) .
$$

Both the detector thickness $(t=50 \mu \mathrm{m})$ and, for a given photon energy, the absorption length ( $\lambda=29 \mu \mathrm{m}$ for $5.9 \mathrm{keV}$ x-rays) are known so the relationship between $z$ and $N_{z_{-} \text {frac }}$ is completely specified.

We also sort the observed events in order of width to form their cumulative distribution as a function of that parameter. Invoking our physically motivated assumption that width decreases with depth, we use this empirical width distribution, together with Eq. (2), to determine the relationship between width and depth. We note that it is important to include all events in this calculation, even the ones with very small measured width, in spite of the fact that the width of such events is not measured accurately, as discussed in Sec. 3.1.

The resulting measurements of event width as a function of depth below the entrance window are shown for different values of $V_{\text {sub }}$ in Fig. 5. As expected, stronger internal fields (more negative $V_{\text {sub }}$ ) reduce the diffusion time and thus event widths. At $V_{\text {sub }}>-3 \mathrm{~V}$, the shape of the curves is different than at lower $V_{\text {sub }}$. This is almost certainly due to formation of an undepleted layer of silicon near the illuminated surface, in which charge collection may be inefficient, excluding some events from our analysis. This would violate our assumption that all events are included and would introduce errors in our assignment of depth to width. This interpretation is supported by the simulated width-depth curves shown in Fig. 3; the simulations do not include such surface effects, and the simulated width-depth curves do not show this shape change.

Another limitation of this algorithm is that it neglects uncertainties in measurements of $\sigma_{\text {spatial }}$. We defer an investigation of this effect to future work. 


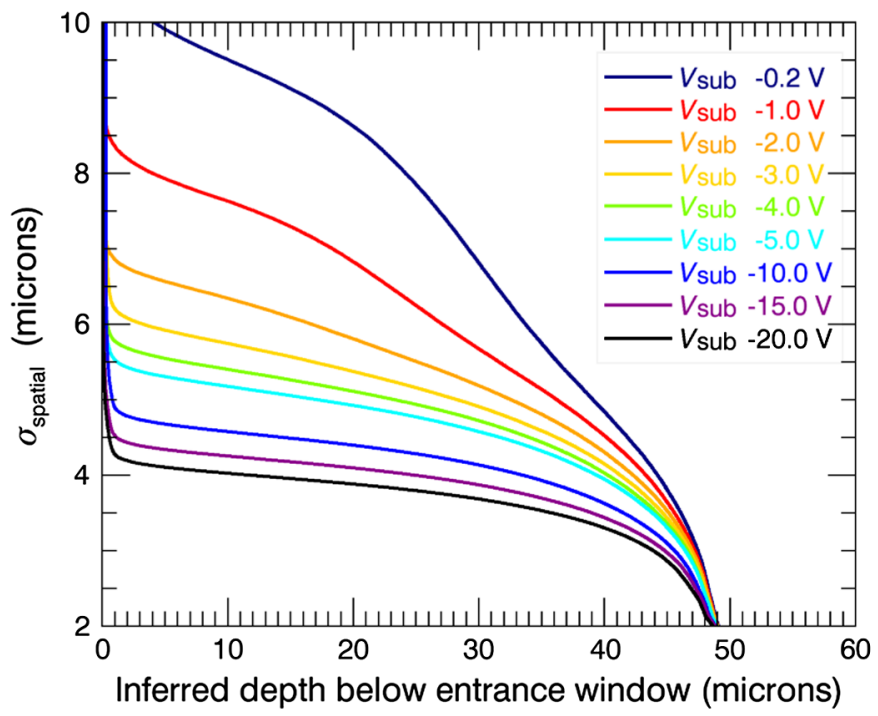

Fig. 5 Measured cumulative distributions of event width $\left(\sigma_{\text {spatial }}\right)$ for $5.9 \mathrm{keV}$ x-rays as a function of absorption distance (depth) below the entrance window.

\subsubsection{Detector characterization from differential event width distributions}

In this section, we turn from the cumulative to the differential form of the width distribution and show how it varies with detector bias. In principle, differential width distributions are an important tool in predicting detector performance. They can be used, for example, to estimate proportions of events with different pixel multiplicities, which in turn are needed to predict the spectral resolution of an image sensor. They also provide powerful observables for use in validating a detector model, especially when measured over a range of internal field conditions.

Figure 6 shows these distributions for $5.9 \mathrm{keV}$ x-rays over a range of substrate bias. The distributions shown include only those events with measurable widths, that is, those for which at least four pixels have measurable charge.

The bias dependence of these distributions not only tells a now familiar story but also provides new insights. At the largest bias ( $V_{\text {sub }}=-20 \mathrm{~V}$, black histogram), the bulk of the silicon is fully depleted. As there is a strong electric field throughout the device, drift times are short, so the corresponding width distribution is relatively narrow and shows a sharp edge at $3.9 \mu \mathrm{m}$. This edge can be interpreted as the width of events interacting in the immediate vicinity of the detector entrance surface. This maximum event width should apply to events of all energies and is thus a

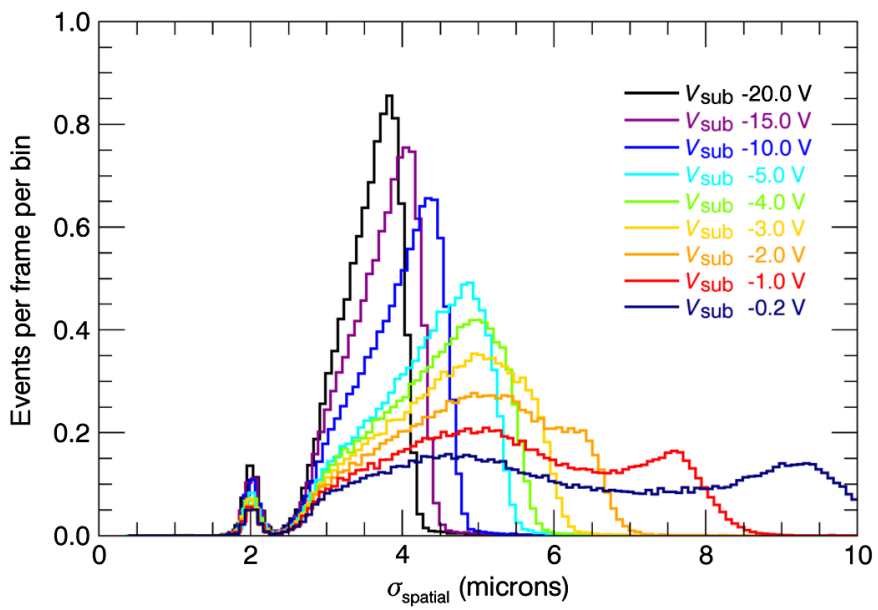

Fig. 6 The differential width distributions of events with pixel multiplicity greater than 3 produced by $5.9 \mathrm{keV}$ x-rays. Distributions for a range of detector bias $\left(V_{\text {sub }}\right)$ conditions are shown. 
very useful parameter for estimating the response of the detector to low energy photons, which are necessarily absorbed there. The shape of the left side of the distribution is determined by the width-depth relation and is thus sensitive to internal field distributions and charge transport properties of the detector. The steep but finite slope of the edge is in principle a measure of the statistical uncertainty of the width measurement. The small peak near $\sigma_{\text {spatial }}=2 \mu \mathrm{m}$ is caused by very narrow events produced close to the buried channel, for which our width measurement is not reliable, as discussed in Sec. 3.1.

As $V_{\text {sub }}$ increases (algebraically), the internal electric field strength drops, broadening the distribution to larger event widths, until a clear transition occurs at $V_{\text {sub }}=-4 \mathrm{~V}$. This is caused by the formation of an undepleted region near the illuminated surface of the device. As internal field strength drops further, the events in the undepleted region form a separate hump in the histogram at much larger widths. In this way, the differential width distributions provide a readily measurable indicator of full depletion within the device. A simple one-dimensional calculation of $V_{\text {sub }}$ at which full depletion of 50- $\mu$ m thick slab of silicon with doping concentration of $2.65 \times 10^{12} \mathrm{~cm}^{-3}$ would occur yields a value of $V_{\text {sub }}=5.1 \mathrm{~V}$, in good agreement with both our experimental and simulated full depletion transition.

Figure 7 compares the event width distributions derived from the simulations with those from the measurements. We tuned the simulation's DiffMultiplier parameter (described in Sec. 2.4) for each $V_{\text {sub }}$ so the peak of the simulated distribution matched the peak of the measured distribution. The tuned values have a small range of 1.4 to 1.7 , with smaller values for smaller (less negative) $V_{\text {sub. }}$. The upper end of this range is not too different from the value DiffMultiplier $\approx 1.9$ expected for a canonical value of the thermal velocity electron effective mass ${ }^{23}$ although it differs noticeably from the value of 2.3 determined for (fully depleted) Vera Rubin Observatory CCDs. ${ }^{18}$ Our simulations for $V_{\text {sub }}<-5 \mathrm{~V}$, at which the device is fully depleted, show good agreement with our data. Agreement is poorer at less negative $V_{\text {sub }}$; the measured distribution for $-0.2 \mathrm{~V}$ has a more extended tail to larger $\sigma_{\text {spatial }}$ than the simulations would predict. This is likely due to both a poor representation of the backside passivation layer and an inadequate correction for backside surface charge losses in the simulation. These issues are exacerbated when the region near the backside is not fully depleted, producing a field-free region where electrons can linger and greatly affecting the measured charge diffusion. Both the need for tuned diffusion and possible improvements to undepleted backside characterization will be addressed in a future paper focused on simulations.

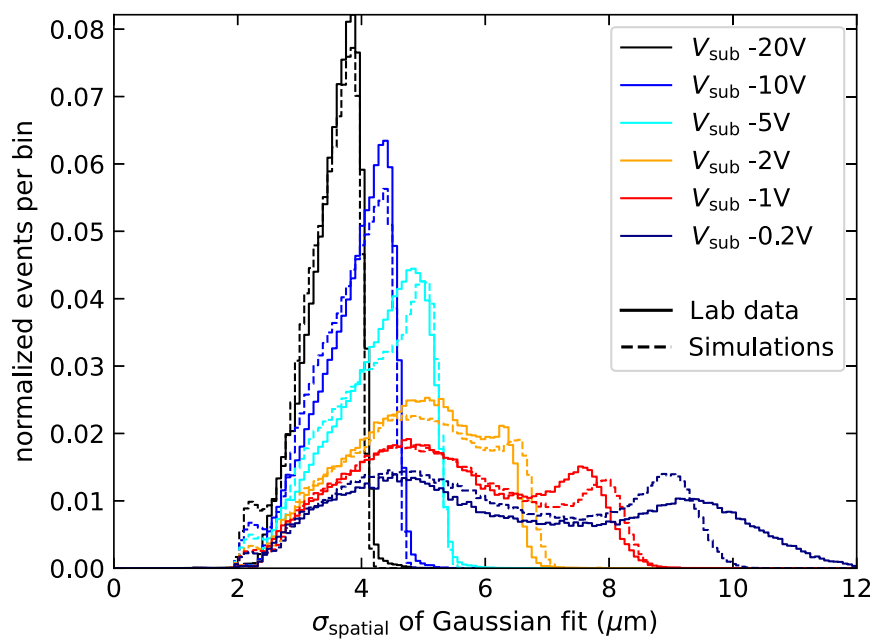

Fig. 7 The distribution of Gaussian $\sigma_{\text {spatial }}$ from simulations of $5.9 \mathrm{keV}$ x-rays, compared with the data (see Fig. 6). Only events with at least 4-pixel multiplicity are shown, and for clarity some $V_{\text {sub }}$ values are not plotted. A single diffusion factor was tuned for each simulation to recover a similar distribution to the data, as described in the text. The simulated distributions are similar to the data for large negative $V_{\text {sub }}$ in which the device is fully depleted. For small $V_{\text {sub }}$ and large $\sigma_{\text {spatial }}$, the distributions differ significantly, likely due to limitations of the simulations. 


\subsection{Event Amplitude Estimation}

The spectral resolution of an x-ray image sensor is its spectral resolution that depends on the accuracy with which the total charge associated with an x-ray event (the event "amplitude") is measured. Traditionally, in large-pixel devices (those with pixels much larger than characteristic event widths) used for Chandra, XMM-Newton, Suzaku, and other X-ray instruments, the amplitude is estimated as the sum signal in a few pixels around a local maximum exceeding the split threshold. In detectors with pixel sizes comparable to characteristic event widths, the majority of events have charge spread over multiple pixels. In this case, numerous pixels that fall below the split threshold may in aggregate contain a significant fraction of the total charge, and it is natural to consider whether alternative methods that explicitly account for finite event width could provide a better estimate of event amplitude.

We evaluated direct fitting of the event charge distribution as a method for determining event amplitude. A comparison of this approach with simple summing of pixels exceeding the split threshold is shown in Fig. 8 for 5.9 and $1.25 \mathrm{keV}$ photons. To investigate the magnitude of charge lost due to the thresholding used in the traditional algorithm, we separately plot spectra for events of various pixel multiplicities. In Fig. 9, we show spectral FWHM and peak location as a

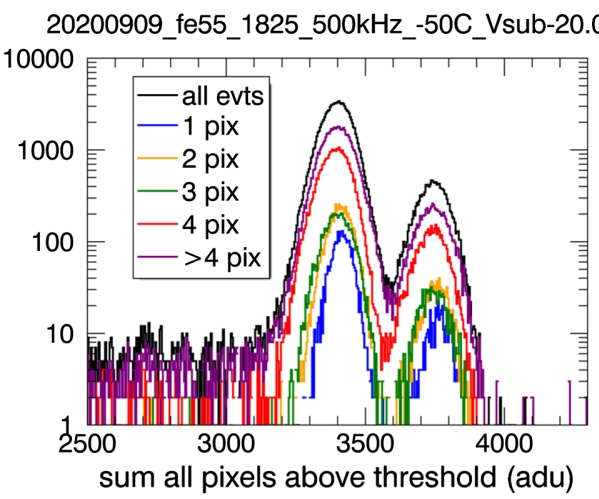

(a)

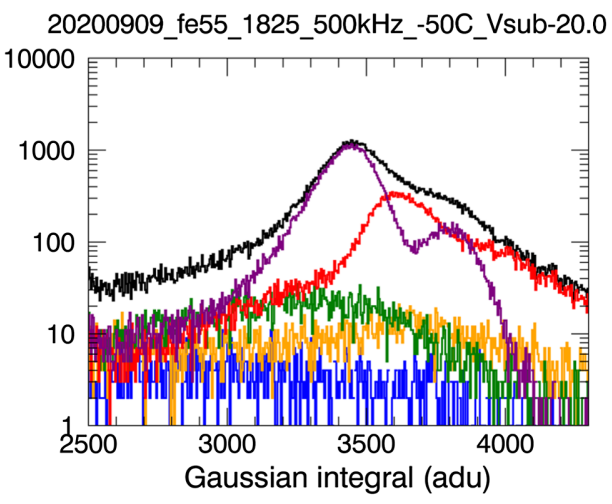

(c)

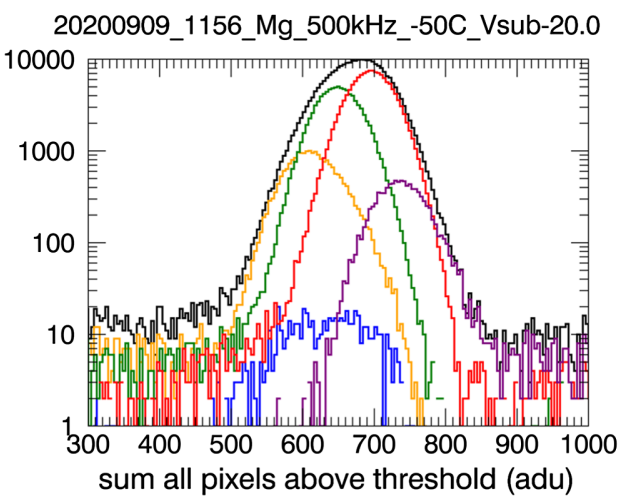

(b)

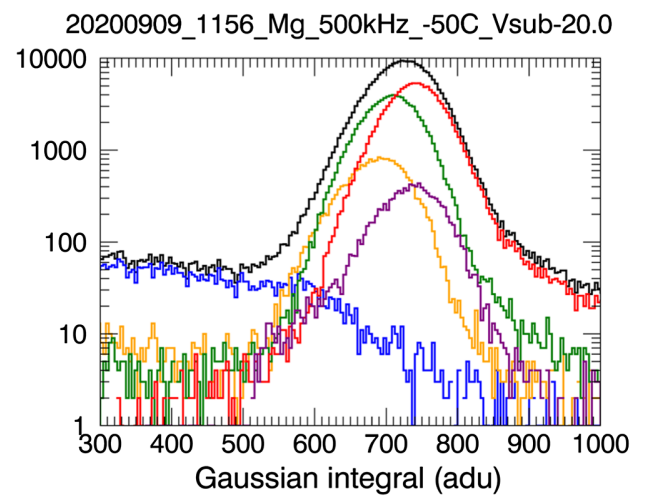

(d)

Fig. 8 Measured event amplitude spectra. Different colors show results for events of various pixel multiplicities. Left panels ( $a$ and c) show results for $5.9 \mathrm{keV}$. Right panels (b and d) show results for $1.25 \mathrm{keV}$. (Top) Event amplitudes are calculated by summing all pixels above split threshold or (bottom) by integrating under the Gaussian fit to the spatial charge distribution of each event. All data were obtained with strong internal electric fields $\left(V_{\text {sub }}=-20 \mathrm{~V}\right)$. At the higher energy, the sum of pixels method produces a much narrower spectral response, although the performance of the Gaussian method can be improved by eliminating events with low multiplicity. At the lower energy, the Gaussian method produces a similar core spectral response compared with the summed pixel method, but with an extended high-energy tail populated predominantly by 4-pixel events. The broad spectra of low-multiplicity events at both energies are caused by poor performance of the Gaussian fit for undersampled distributions. 

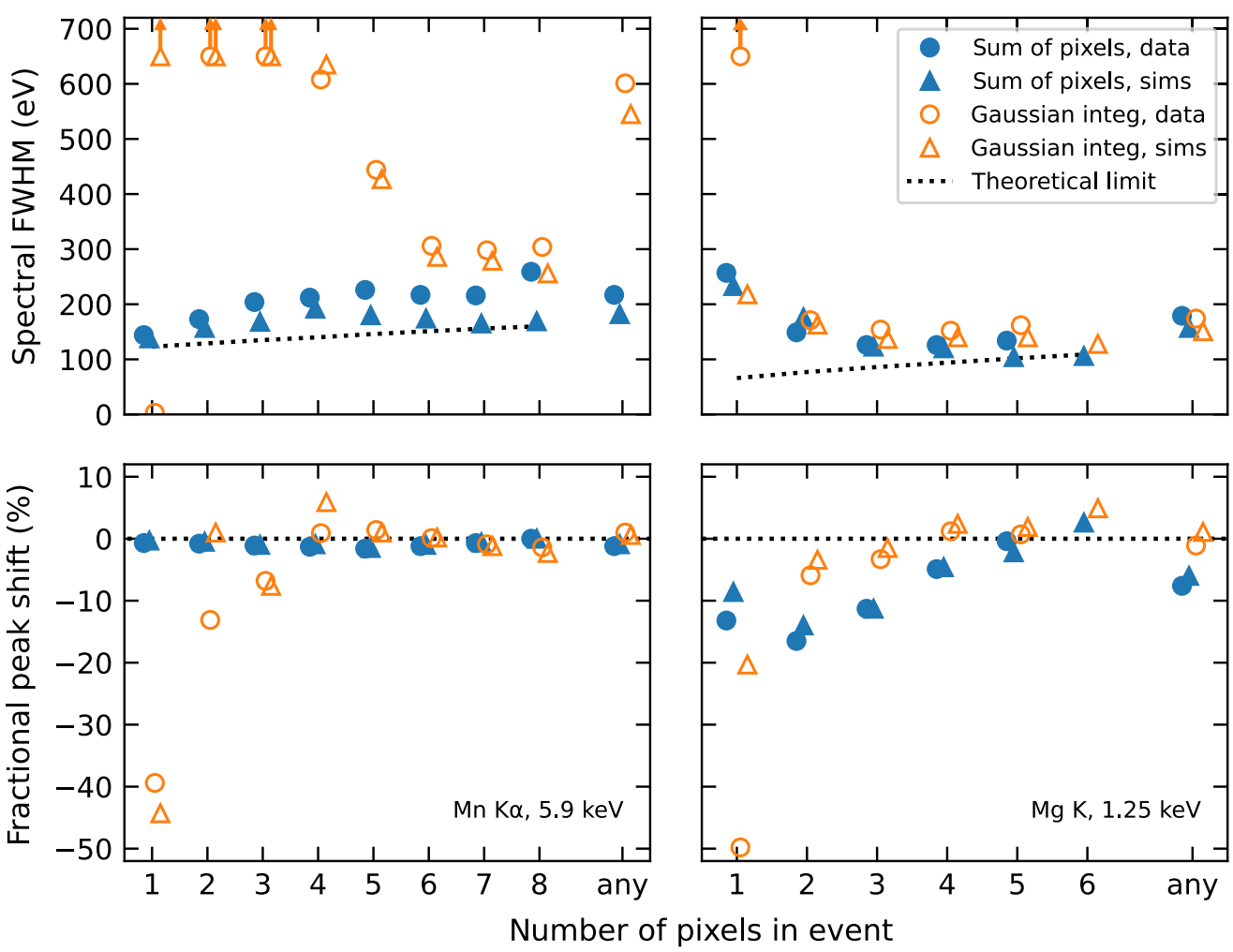

Fig. 9 (Top) Spectral response parameters FWHM and (bottom) peak shift for (left) 5.9 keV and (right) $1.25 \mathrm{keV}$. These were measured by fitting a single Gaussian to the spectra shown in Figs. 8 and 10. Points with arrows indicate values out of the plot range. The spectral FWHM "theoretical limit" is the Fano spectral width convolved with pixel-based noise of 4.5 electrons RMS. At $5.9 \mathrm{keV}$, the spatial Gaussian integral summation method performs poorly compared with a simple sum of pixel values, except when the number of pixels above threshold is large. At $1.25 \mathrm{keV}$, the spatial Gaussian performs similarly or better than the pixel summation method. See Tables 2 and 3 in Appendix A for a full tabulation of values.

function of pixel multiplicity (see also Appendix A). These data were obtained with substrate bias $V_{\text {sub }}=-20 \mathrm{~V}$, providing the maximum internal electric field strength. In general, integrating the best-fit spatial Gaussian does produce a slightly higher estimate for the event amplitude, consistent with the idea that a functional form accurately describing the event shape can recover signal lost to the surrounding pixels that fall below split threshold. On the other hand, the spectral distributions derived from the Gaussian fits are noticeably broader and themselves clearly nonGaussian, especially at $5.9 \mathrm{keV}$.

The 5.9-keV spectra in the left panels of Fig. 8 and the corresponding response parameters in the left panels of Fig. 9 show that the Gaussian fit performs worst for events for which a relatively small number of pixels (roughly 5 or fewer) exceed the split threshold. We attribute this behavior to the spatial undersampling of events with intrinsically narrow charge distributions. We also note that at this energy there is only a small change in the spectral peak location with pixel multiplicity for either amplitude determination method, suggesting that relatively little charge is contained in pixels below the split threshold.

The situation is different at the lower energy, as the right panels of Figs. 8 and 9 show. Here, the performance of the two amplitude determination methods is quite similar, and spectral widths are in some cases marginally better for Gaussian fits. Remarkably, good results are obtained with this method even for events with as few as two pixels above threshold, suggesting that as expected these events are more extended and suffer less from undersampling than their counterparts at higher energy. The low pixel multiplicity of these events is due to truncation by the threshold rather than an intrinsically narrow spatial distribution. This interpretation also explains the systematic increase of spectral peak location with pixel multiplicity at this energy. Figure 9 shows that Gaussian fits are indeed less susceptible, though not immune, to spectral broadening 


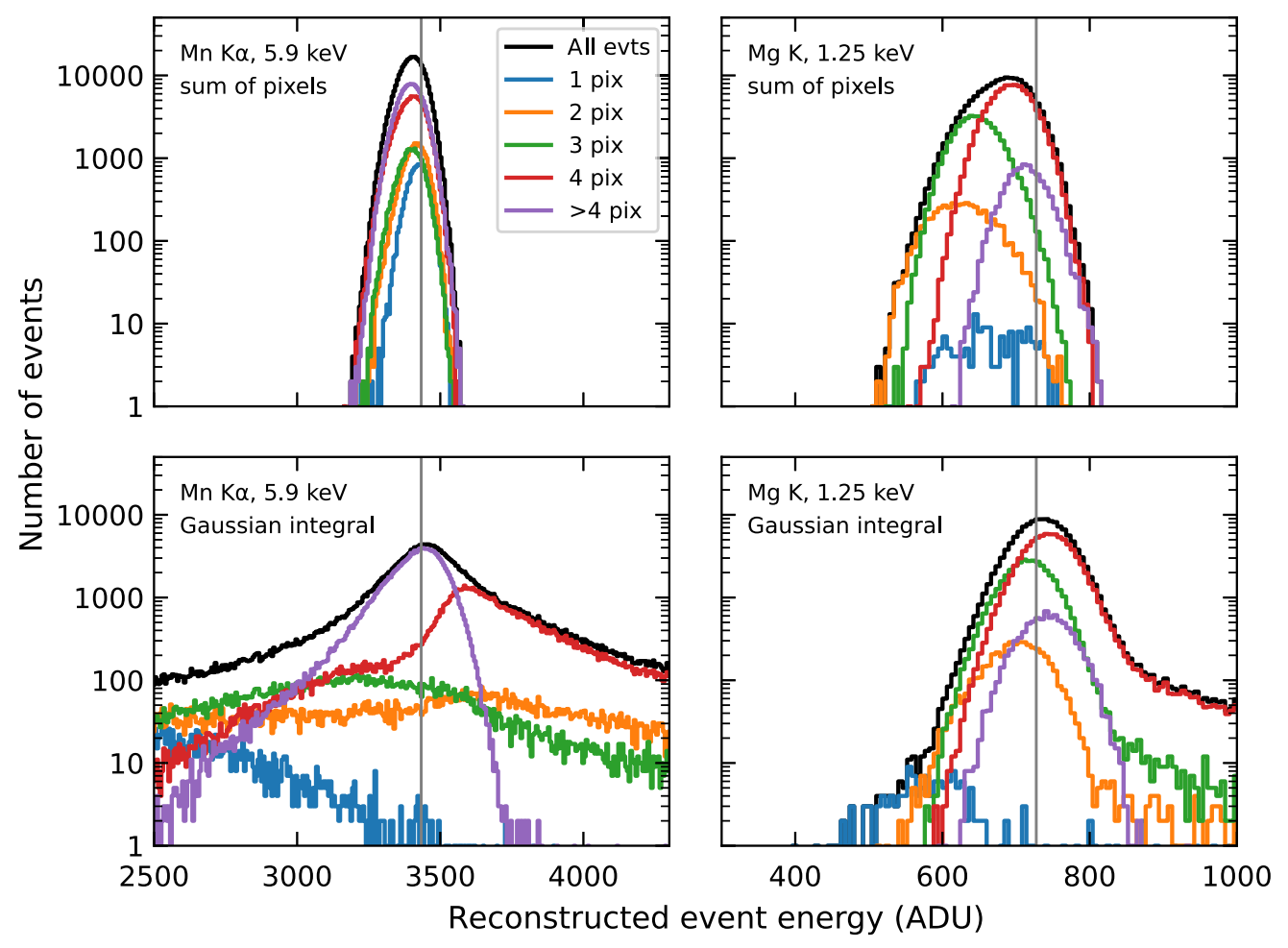

Fig. 10 Spectra of simulated events from (left) $5.9 \mathrm{keV}$ photons and (right) $1.25 \mathrm{keV}$ photons, with the event energies calculated (top) by summing all pixels above split threshold and (bottom) by integrating under a Gaussian fit. Spectra are separated by the number of pixels above the split threshold, as in Fig. 8. At both energies, the Gaussian integral method performs better at estimating the most likely energy of the ensemble of events, as the peak is closer to the expected energy indicated by the vertical line. The effects of few-pixel events are similar to those shown in Fig. 8 and are caused by poor performance of the Gaussian fit for undersampled distributions.

due to charge loss at this energy: peak locations change less with pixel multiplicity, and, as a result, the spectral full-width at half-maximum for the entire data set is actually slightly better for the fitting method than for pixel summation.

A complication of this simple picture is presented by the spectrum of four-pixel events derived from Gaussian fitting, which shows a high-energy tail. We interpret this tail as another consequence of poor fitting arising from (maximal) undersampling of those charge clouds with spatial centroids close to the center of a $2 \times 2$ pixel array. Examination of the fitted centroids of these tail events confirms this interpretation. We defer a detailed analysis of photon location to future work.

These inferences are generally supported by simulations, although the details are subtle, as shown in Fig. 10. Here again, we separately plot spectra for events with different pixel multiplicities. The results are remarkably similar to the measured data. At both energies, the Gaussian fitting method does indeed improve the amplitude estimate, the true value of which is known from the simulation inputs; the peaks of the spectral distributions are very close to the input energy. At $5.9 \mathrm{keV}$, the simulated spectral redistribution is much broader using the Gaussian method than the sum of pixels, just as we observe in the measured spectra. These broad features are dominated by events with low pixel multiplicities. At $1.25 \mathrm{keV}$, the Gaussian fit and pixel summing estimates produce similar core spectral responses, whereas the Gaussian fit estimate features an extended high-energy tail populated mainly by four-pixel events.

In summary, we find that for our devices and at the energies we probed, although a Gaussian fit to individual events may provide a slightly less biased amplitude estimate on average, the effective spectral resolution is worse at $5.9 \mathrm{keV}$ and comparable at $1.25 \mathrm{keV}$ to that obtained with the traditional sum of pixels above threshold algorithm. At the higher energy, the spatial sampling provided by $8 \mu \mathrm{m}$ pixels is generally insufficient to support spatial modeling of 
individual events. At $1.25 \mathrm{keV}$, the fitting method recovers some of the subthreshold signal ignored by the pixel summation algorithm. This results in event amplitude estimates with less bias, but with no less dispersion, than those of the summation method.

\section{Summary and Discussion}

Our measurements of x-ray-induced charge packets have yielded a number of results. We confirm that charge packets produced at a given detector depth exhibit (on average) a Gaussian spatial distribution to remarkable accuracy. We show that the distribution of charge packet widths, parameterized by the Gaussian standard deviation $\sigma_{\text {spatial }}$, provides useful information on detector structure. The cumulative width distribution gives the relationship between width and interaction depth. The differential width distribution provides, for a fully depleted detector, a precise estimate of the size of events produced in the immediate vicinity of the entrance window. Remarkably, this parameter, which is a crucial determinant of detector response to low-energy $(<1 \mathrm{keV}) \mathrm{x}$-rays, is most easily and accurately measured with higher energy $\mathrm{x}$-rays using the techniques we present here. The shape of the differential width distribution also provides a clear indication of the extent to which the detector is fully depleted.

We have used these diagnostics to tune and validate an implementation of the Poisson CCD simulation. We find reasonable agreement with measurements by reducing the amount of (lateral) diffusion, relative to values reported for VRO devices, ${ }^{18}$ by of order $35 \%$ when the detector is fully depleted. The poorer agreement for partially depleted configurations indicates that further development of the simulation is needed. We will report on this in a future contribution.

We investigated use of Gaussian fits to individual events to estimate their amplitudes. We found that at $5.9 \mathrm{keV}$ this estimator is not as accurate as traditional methods that sum pixel values exceeding a threshold. For a significant fraction of events at this energy, our detector provides insufficient spatial resolution to measure event shape. At $1.25 \mathrm{keV}$, the fitting method clearly recovers signal that is neglected by traditional pixel summation algorithms. While this produces a less biased amplitude estimate, it does not improve spectral resolution at the energies we have investigated. This may be a consequence of the relatively low signal-to-noise ratio of the subthreshold pixels included in the fits. We speculate that more sophisticated fitting algorithms, for example, incorporating priors based on readily measurable event characteristics, may be more successful. We shall also explore this approach in future work.

Finally, our results highlight an important and perhaps underappreciated mechanism through which read noise can degrade spectral resolution at lower x-ray energies. It is widely understood that spectral resolution is degraded when charge is shared among multiple pixels since the readout noise associated with each pixel sums in quadrature in the event amplitude calculation. We have shown the importance of a second mechanism by which readout noise degrades spectral resolution; the effective loss of signal in pixels with values below threshold (see the right-hand panels of Fig. 8). Since the threshold must be a multiple of readout noise, the magnitude of this lost subthreshold signal increases as readout noise increases. In fact, the spectral broadening due to lost, subthreshold charge in our $1.25 \mathrm{keV}$ data is considerably larger than that due to the noise injected by the sense node amplifier itself, as is demonstrated in the right-hand panels of Fig. 9. The spectral resolution is much worse than the theoretical (Fano plus readnoise) expectation, and the charge lost is largest (peak shift $10 \%$ to $20 \%$ ) for pixel multiplicities $n=1$ and $n=2$. As a result, the integrated spectral resolution, summed over all events $(\sim 150 \mathrm{eV}$ FWHM $)$ is considerably worse than expected from Fano noise plus the weighted quadrature sum of readout noise alone $(<90 \mathrm{eV}$ FWHM.)

\section{Conclusions and Future Work}

Mega-pixel x-ray sensors with large ratios of depletion thickness to pixel size are required for future strategic missions such as Lynx and AXIS. We find that direct charge-cloud size measurements in a 50- $\mu \mathrm{m}$ thick, $8-\mu \mathrm{m}$-pixel device are useful for validating a basic drift and diffusion simulation of such devices, although more work is required to achieve accurate modeling over a wide range of operating conditions. Our measurements and simulations suggest that readnoise dependent, subthreshold charge loss may be the most important determinant of low-energy

J. Astron. Telesc. Instrum. Syst. $\quad$ 016004-15 Jan-Mar 2022 • Vol. 8(1) 
spectral resolution, and it is therefore essential that this process is fully understood when establishing sensor noise requirements for these missions. In fact, the detector model described here predicts that sensors capable of meeting the low-energy spectral resolution requirements of AXIS and Lynx require noise considerably below their notional upper limit of four electrons RMS.

To test this proposition and to make it quantitative, we are extending this work in several ways. We are currently acquiring data at lower x-ray energies with lower noise detectors. These data will provide more stringent tests of the current simulation. We are also working to improve the fidelity of the simulation by refining the treatment of lateral diffusion and incorporating a more realistic model of the detector entrance window. We expect this work to lead to more robust detector requirements for future $\mathrm{x}$-ray missions.

\section{Appendix A: Tabulation of Spectral Response Parameters}

We include here in Tables 2 and 3 tabulation of the spectral response parameters plotted in Fig. 9 for different event pixel multiplicities, as discussed in Sec. 3.3. The spectra FWHM and peak shift are measured from fitting a single Gaussian to the spectra shown in Figs. 8 and 10.

Table 2 Spectral resolution using different event reconstruction methods.

\begin{tabular}{|c|c|c|c|c|c|c|}
\hline \multirow[b]{3}{*}{ Energy } & \multirow[b]{3}{*}{ \# pix } & \multirow{3}{*}{$\frac{\text { Fano }+ \text { noise }^{\mathrm{a}}}{\text { FWHM }(\mathrm{eV})}$} & \multicolumn{2}{|c|}{ Measurements $^{\mathrm{b}}$} & \multicolumn{2}{|c|}{ Simulations $^{b}$} \\
\hline & & & Pixel sum & Gauss. int. & Pixel sum & Gauss. int. \\
\hline & & & FWHM (eV) & FWHM (eV) & FWHM (eV) & $\mathrm{FWHM}(\mathrm{eV})$ \\
\hline \multirow[t]{9}{*}{$5.9 \mathrm{keV}$} & Any & $\ldots$ & 217 & 601 & 182 & 545 \\
\hline & 1 & 123 & 144 & 3 & 138 & 1120 \\
\hline & 2 & 129 & 173 & 6030 & 157 & 4044 \\
\hline & 3 & 135 & 204 & 1784 & 168 & 1701 \\
\hline & 4 & 140 & 212 & 608 & 192 & 635 \\
\hline & 5 & 146 & 226 & 444 & 180 & 427 \\
\hline & 6 & 151 & 217 & 306 & 174 & 286 \\
\hline & 7 & 156 & 216 & 298 & 165 & 279 \\
\hline & 8 & 160 & 259 & 304 & 169 & 256 \\
\hline \multirow[t]{7}{*}{$1.25 \mathrm{keV}$} & Any & $\ldots$ & 179 & 174 & 157 & 151 \\
\hline & 1 & 66 & 257 & 758 & 233 & 218 \\
\hline & 2 & 77 & 149 & 171 & 176 & 163 \\
\hline & 3 & 86 & 126 & 154 & 123 & 137 \\
\hline & 4 & 94 & 126 & 152 & 120 & 140 \\
\hline & 5 & 102 & 134 & 162 & 104 & 140 \\
\hline & 6 & 109 & $\ldots$ & $\ldots$ & 106 & 128 \\
\hline
\end{tabular}

a"Fano + noise" is the theoretical Fano-limit spectral FWHM added in quadrature with Gaussian readout noise in each pixel.

${ }^{b}$ Conversion from measured analog-to-digital units (ADU) to $\mathrm{eV}$ uses a gain factor derived from the peak of the $5.9 \mathrm{keV}$ histogram of events with pixel multiplicity $\geq 5$ [see Fig. 8(a)]. The simulations assume an electron liberation energy of $3.65 \mathrm{eV}$ to convert to energy, and the same measured gain factor of $1.718 \mathrm{eV} \mathrm{ADU}^{-1}$ to compare to lab data ADU values. 
Table 3 Spectral peak shift ${ }^{\mathrm{a}}$ using different event reconstruction methods.

\begin{tabular}{|c|c|c|c|c|c|c|c|}
\hline \multirow[b]{2}{*}{ Energy } & \multirow[b]{2}{*}{ \# pix } & \multirow[b]{2}{*}{$\begin{array}{l}\text { Frac. } \\
\text { events }\end{array}$} & \multicolumn{3}{|c|}{ Measurements } & \multicolumn{2}{|c|}{ Simulations } \\
\hline & & & $\begin{array}{c}\text { Pixel sum } \\
\text { peak shift (\%) }\end{array}$ & $\begin{array}{c}\text { Gauss. int. } \\
\text { peak shift (\%) }\end{array}$ & $\begin{array}{l}\text { Frac. } \\
\text { events }\end{array}$ & $\begin{array}{c}\text { Pixel sum } \\
\text { peak shift (\%) }\end{array}$ & $\begin{array}{c}\text { Gauss. int. } \\
\text { peak shift (\%) }\end{array}$ \\
\hline \multirow[t]{9}{*}{$5.9 \mathrm{keV}$} & Any & 1 & -1.2 & +1.0 & 1 & -0.9 & +0.6 \\
\hline & 1 & 0.02 & -0.7 & -39.4 & 0.04 & -0.3 & -44.3 \\
\hline & 2 & 0.06 & -0.8 & -13.1 & 0.08 & -0.5 & +1.0 \\
\hline & 3 & 0.06 & -1.1 & -6.8 & 0.0 & -1.0 & -7.6 \\
\hline & 4 & 0.30 & -1.3 & +0.9 & 0.35 & -0.9 & +5.9 \\
\hline & 5 & 0.16 & -1.6 & +1.4 & 0.14 & -1.5 & +1.0 \\
\hline & 6 & 0.25 & -1.2 & +0.1 & 0.22 & -1.0 & +0.2 \\
\hline & 7 & 0.13 & -0.7 & -0.9 & 0.09 & -0.6 & -1.2 \\
\hline & 8 & 0.02 & +0.0 & -1.4 & 0.004 & +0.0 & -2.3 \\
\hline \multirow[t]{7}{*}{$1.25 \mathrm{keV}$} & Any & 1 & -7.6 & -1.1 & 1 & -6.0 & +1.1 \\
\hline & 1 & 0.002 & -13.2 & -49.8 & 0.001 & -8.6 & -20.3 \\
\hline & 2 & 0.082 & -16.5 & -5.9 & 0.03 & -14.0 & -3.4 \\
\hline & 3 & 0.353 & -11.3 & -3.3 & 0.27 & -11.3 & -1.5 \\
\hline & 4 & 0.526 & -4.9 & +1.2 & 0.63 & -4.6 & +2.4 \\
\hline & 5 & 0.034 & -0.4 & +0.7 & 0.06 & -2.2 & +1.9 \\
\hline & 6 & 0.000 & $\ldots$ & $\cdots$ & 0.002 & +2.6 & +4.9 \\
\hline
\end{tabular}

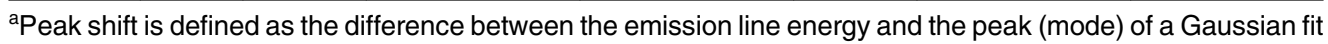
to the spectrum.

\section{Acknowledgments}

We are deeply saddened by the passing of our co-author and colleague Barry E. Burke. His work advanced imaging technology and enabled the contributions of generations of astronomers to scientific knowledge. He was a most generous and, in the very highest sense, a good human being. We thank Michelle Gabutti for assistance with data acquisition, Craig Lage for providing key updates to and generous assistance with the POISSON CCD code, and Sven Herrmann for valuable discussions. We gratefully acknowledge support of this work by NASA through Strategic Astrophysics Technology Grant Nos. 80NSSC18K0138 and 80NSSC19K0401 to MIT and by MKI's Kavli Research Infrastructure Fund.

\section{References}

1. B. Burke et al., "CCD soft x-ray imaging spectrometer for the ASCA satellite," IEEE Trans. Nucl. Sci. 41, 375-385 (1994).

2. G. P. Garmire et al., "Advanced CCD imaging spectrometer (ACIS) instrument on the Chandra x-ray Observatory," Proc. SPIE 4851, 28-44 (2003).

3. F. Jansen et al., "XMM-Newton observatory. I. The spacecraft and operations," Astron. Astrophys. 365, L1-L6 (2001).

4. N. Gehrels et al., "The swift gamma-ray burst mission," Astrophys. J. 611, 1005-1020 (2004). 
5. K. Mitsuda et al., "The x-ray observatory Suzaku," Publ. Astron. Soc. Jpn. 59, S1-S7 (2007).

6. P. Predehl et al., "The eROSITA x-ray telescope on SRG," Astron. Astrophys. 647, A1 (2021).

7. J. A. Gaskin et al., "Lynx X-ray Observatory: an overview," J. Astron. Telesc. Instrum. Syst. 5, 021001 (2019).

8. R. Mushotzky, "AXIS: a probe class next generation high angular resolution x-ray imaging satellite," Proc. SPIE 10699, 1069929 (2018).

9. D. E. Groom et al., "Back-illuminated, fully-depleted CCD image sensors for use in optical and near-IR astronomy," Nucl. Instrum. Methods Phys. Res. A 442, 216-222 (2000).

10. F. W. High et al., "Pixelation effects in weak lensing," Publ. Astron. Soc. Pac. 119, 12951307 (2007).

11. H. Marshall et al., "A small satellite version of a broad-band soft x-ray polarimeter," Proc. SPIE 11444, 114442Y (2020).

12. M. Schattenburg et al., "A diffraction limited Wolter nested-shell telescope concept with Pico-radian resolution," Proc. SPIE 11444, 114441F (2020).

13. M. W. Bautz et al., "Toward fast, low-noise charge-coupled devices for Lynx," J. Astron. Telesc. Instrum. Syst. 5, 021015 (2019).

14. A. D. Falcone et al., "Overview of the high-definition x-ray imager instrument on the Lynx x-ray surveyor," J. Astron. Telesc. Instrum. Syst. 5, 021019 (2019).

15. S. V. Hull et al., "Hybrid CMOS detectors for the Lynx x-ray surveyor high definition x-ray imager," J. Astron. Telesc. Instrum. Syst. 5, 021018 (2019).

16. A. Kenter, R. Kraft, and T. Gauron, "Monolithic CMOS detectors for use as x-ray imaging spectrometers," Proc. SPIE 11118, 1111806 (2019).

17. S. Herrmann et al., "MCRC V1: development of integrated readout electronics for next generation x-ray CCD detectors for future satellite observatories," Proc. SPIE 11454, 1145427 (2020).

18. C. Lage et al., "Poisson_CCD: a dedicated simulator for modeling CCDs," J. Appl. Phys. 130, 164502 (2021).

19. Ž. Ivezić et al., "LSST: from science drivers to reference design and anticipated data products," Astrophys. J. 873, 111 (2019).

20. I. V. Kotov et al., "X-ray analysis of fully depleted CCDs with small pixel size," Nucl. Instrum. Methods Phys. Res. A 787, 12-19 (2015).

21. G. Bredthauer, "Archon: a modern controller for high performance astronomical CCDs," Proc. SPIE 9147, 91475B (2014).

22. C. B. Markwardt, "Non-linear least-squares fitting in IDL with MPFIT," Astron. Soc. Pac. Conf. Ser. 411, 251 (2009).

23. M. A. Green, "Intrinsic concentration, effective densities of states, and effective mass in silicon," J. Appl. Phys. 67(6), 2944-2954 (1990).

24. M. S. Haro, G. Fernandez Moroni, and J. Tiffenberg, "Studies on small charge packet transport in high-resistivity fully depleted CCDs," IEEE Trans. Electron Devices 67(5), 1993-2000 (2020).

25. I. Prigozhin, S. Dominici, and E. Bellotti, "FBMC3D: a large-scale 3-D Monte Carlo simulation tool for modern electronic devices," IEEE Trans. Electron Devices 68(1), 279-287 (2021).

26. G. Prigozhin et al., "An experimental study of charge diffusion in the undepleted silicon of x-ray CCDs," IEEE Trans. Electron Devices 50, 246-253 (2003).

27. E. Miyata et al., "Application of the Mesh experiment for the back-illuminated chargecoupled device: I. Experiment and the charge cloud shape," Jpn. J. Appl. Phys. 41, 5827 (2002).

28. G. G. Pavlov and J. A. Nousek, "Charge diffusion in CCD x-ray detectors," Nucl. Instrum. Methods Phys. Res. A 428, 348-366 (1999).

29. I. V. Kotov et al., "Analysis of the EMCCD point-source response using x-rays," Nucl. Instrum. Methods Phys. Res. A 985, 164706 (2021).

Beverly J. LaMarr is sponsored research technical staff at the MIT Kavli Institute for Astrophysics and Space Research. She received her BS degree in electrical engineering from 
Hofstra University, New York, in 1992. She supports laboratory testing and characterization of experimental x-ray detectors. She also supports Advanced CCD Imaging Spectrometer on NASA's Chandra X-ray Observatory and the X-ray Timing Instrument on the Neutron Star Interior Composition ExploreR.

Eric D. Miller is a research scientist at the MIT Kavli Institute for Astrophysics and Space Research. He received his BA degree in physics from Oberlin College in 1996, and his MS and $\mathrm{PhD}$ degrees in astronomy and astrophysics from the University of Michigan in 1998 and 2003, respectively. He leads the XRISM In-Flight Calibration Planning Team, develops X-ray imaging detectors for future missions, and studies galaxy clusters and the diffuse intergalactic medium.

Catherine E. Grant received her bachelor's degree in astronomy and astrophysics from Harvard University in 1993 and her $\mathrm{PhD}$ in astronomy and astrophysics from Penn State University in 1999. She is currently a research scientist at the MIT Kavli Institute for Astrophysics and Space Research. She as an instrument scientist for ACIS on the Chandra X-ray Observatory, he has had roles in on-orbit performance monitoring, ground and flight calibration, and operation of the CCD camera.

Biographies of the other authors are not available. 